\title{
LAS ELECCIONES AL PARLAMENTO ANDALUZ *
}

El día 23 de mayo de 1982 se celebraron las primeras elecciones al Parlamento Andaluz. De esta manera, Andalucía se convierte en la cuarta y última de las comunidades que alcanzaron su autonomía por la vía del artículo 151 de la Constitución en dotarse de un cuerpo legislativo autónomo surgido del sufragio universal.

Los pronósticos y sondeos preelectorales de opinión, que auguraban una victoria socialista en estos comicios, se vieron ampliamente rebasados al alcanzar el PSOE la mayoría absoluta de los votos. Si bien es cierto que Andalucía era y es considerada un feudo de la izquierda socialista y comunista, el PSOE ha acaparado la inmensa mayoría de los sufragios de la izquierda andaluza, convirtiéndose en la primera fuerza que consigue la mayoría absoluta en nuestro país desde el comienzo de la transición de la dictadura franquista a la democracia parlamentaria.

El pueblo andaluz, que conquistó su derecho a acceder a la autonomía a través del artículo 151 de la Constitución el día 28 de febreto de 1980 y ratificó en referéndum su Estatuto de Autonomía - llamado de Carmona- el 20 de octubre de 1981, ha elegido un Parlamento que tendrá que hacer frente a todos los graves problemas - muchos de ellos ancestrales - de esta comunidad, que representa el 20 por 100 del territorio del Estado. Una comunidad en la que el paro agrícola e industrial alcanzan sus más altas cotas; una comunidad - patria del latifundioque reclama con carácter de urgencia una solución al problema del reparto de las tierras. Andalucía, que es la zona geográfica que arroja las mayores tasas de emigración, es también la región española donde las desigualdades sociales alcanzan su máxima expresión.

La importancia de estas elecciones venía dada, entre otras causas, por tratarse de las últimas elecciones autonómicas antes de las generales de 1983, y este dato, teniendo en cuenta los cambios acaecidos en el espectro político nacional desde las generales de 1979, redoblaba la expectación ante esta consulta.

La situación política nacional era, a grandes rasgos, la siguiente:

A la derecha, el partido del Gobierno, UCD, que a lo largo de la presente legislatura ha venido sufriendo las crisis más profundas desde su fundación. La gestión del partido gubernamental ha arrojado saldos negativos en las elecciones

* Los cuadros de datos incluidos en este artículo han sido confeccionados en base a los últimos avances provisionales de los resultados de las elecciones al Parlamento Andaluz proporcionados por el Ministerio del Interior. A la hora de cerrarse el presente número de la Revista aún no habían sido hechos públicos los datos definitivos oficiales. 
autonómicas - Cataluña, País Vasco y Galicia-, celebradas con anterioridad a las que en esta ocasión comento. En Cataluña y el País Vasco fueron los partidos de inspiración nacionalista ( $\mathrm{CiU}, \mathrm{PNV}, \mathrm{HB}$ ) los depositarios de la confianza mayoritaria del pueblo, seguidos en ambos casos por el PSOE y, más de lejos, por UCD. Pero fueron los resultados de las elecciones gallegas los que vinieron a introducir un nuevo factor de intranquilidad, por lo distinto de su naturaleza, en el partido del presidente Calvo Sotelo. La victoria de Alianza Popular en Galicia y los posteriores augurios cargados de triunfalismo de Manuel Fraga pronosticando una repetición casi mecánica de los resultados gallegos en futuras consultas electorales, no podían resultar otra cosa que un aviso, un serio toque de atención para el partido centrista. De cualquier forma, un partido conservador venía a arrebatarle votos conservadores a una UCD coyunturalmente conservadora.

Por otro lado, UCD ha padecido convulsiones internas de importancia a lo largo de la actual legislatura, que se han materializado, por una parte, en la escisión protagonizada por el sector situado a la izquierda del partido, esto es, el sector socialdemócrata agrupado en torno a Francisco Fernández Ordóñez -posteriormente convertido en Partido de Acción Democrática-, y por otra, en el abandono del partido por parte de alguno de sus diputados más conservadores y su posterior ingreso en el grupo parlamentario de Coalición Democrática.

Más a la derecha, Alianza Popular ha combinado durante esta legislatura el lanzamiento de la idea de la construcción de una «mayoría natural» que llevaría a la derecha a convertirse en mayoría parlamentaria frente a los «partidos marxistas», con el aprovechamiento en beneficio propio de las crisis internas del partido gubernamental. A raíz de su victoria en las elecciones al Parlamento Gallego, el partido aliancista ha redoblado su empuje y optimismo, presentándose como una fuerza política en alza.

En el terreno de la izquierda, el PSOE continúa ofreciéndose a la opinión pública española como firme alternativa de gobierno. La moderación es la tónica general en el tratamiento de los temas que podrían presentarse como más conflictivos, tanto de índole interna -relaciones con la patronal, nacionalizaciones, proceso autonómico (LOAPA), etc.- como externa -entrada de España en la NATO, etc.- de cara a un próximo acceso al poder de los socialistas de Felipe González.

Más a la izquierda, la aguda crisis arrastrada por el PCE amenaza con convertir al partido eurocomunista de Santiago Carrillo en una formación meramente testimonial de la izquierda española. Así, además de registrar una creciente desafiliación tanto en los sectores de la izquierda como de la derecha de su militancia, sufre constantes amenazas de escisión precisamente en sus zonas de mayor implantación. El caso más claro en este sentido lo constituye la creciente creación del Partido de los Comunistas de Cataluña (PCC), que ha supuesto para el Partido Socialista Unificado de Cataluña (PSUC) la pérdida de más de la mitad de su afiliación.

\section{LA CAMPAÑA}

La campaña previa a la convocatoria del 23 de mayo reunió en Andalucía a las direcciones de todas las fuerzas políticas de ámbito estatal. El Partido Socialista de Andalucía (PSA-PA), que era la única organización de carácter nacionalista en concurrir a las urnas tras la decisión del ex ministro Clavero - líder de Unidad Andaluza, partido recientemente constituido- de no acudir a la confrontación electoral, trató de difundir este mensaje en su campaña. La derecha utilizó toda su fuerza propagandística en el sentido de transmitir al electorado andaluz 
los peligros de una victoria de la izquierda. Los líderes de UCD y AP —en mayor medida los del primer partido- defendieron a sus respectivos candidatos en una fuerte campaña a la contra, denunciando los supuestos intentos de formación de un gobierno con participación comunista tras las elecciones autonómicas, tratando de desviar el voto socialista moderado hacia sus posiciones ideológicas. El argumento más empleado en este sentido fue la reciente inclusión de un comunista en el gobierno socialista del Principado de Asturias. A esta campaña se unió la Confederación de Empresarios Andaluces (CEA), rama regional de la Confederación Española de Organizaciones Empresariales (CEOE). E1 contenido de la propaganda utilizada por la patronal andaluza fue de tal virulencia que, a posteriori, llegó a ser considerada contraproducente. Los lemas más profusamente difundidos fueron: «Andaluz, no dejes que te metan en un puño» (UCD) y «EI trabajo es lo que cuenta» (AP).

La moderación fue la tónica general de la campaña electoral de la izquierda, más segura de su victoria siendo Andalucía su feudo histórico, como ya indiqué al comienzo de este artículo. Frente a las acusaciones de «frentepopulismo», los líderes y candidatos socialistas no dejaron de incidir en que, en caso de una previsible victoria del PSOE, y fueran cuales fueran las condiciones de ésta, no se daría cabida a los comunistas en el gobierno andaluz. El PCA (Partido Comunista de Andalucía), no obstante, basó su campaña en un intento de demostrar la inevitabilidad de su participación gubernamental. Los lemas utilizados: «A la hora de la verdad, vota PSOE de Andalucía» (PSOE) y «Juntos podemos» (PCA).

Los candidatos a la presidencia de la Junta de Andalucía por cada una de las grandes formaciones políticas fueron los siguientes:

- UCD: Luis Merino, abogado, cabeza de lista por la provincia de Málaga.

- PSOE: Rafael Escuredo, presidente saliente de la Junta de Andalucía.

- PCA: Felipe Alcaraz, profesor de Universidad y secretario general de los comunistas andaluces.

- AP: Presentaría como candidato al cabeza de la lista más votada de su partido.

- PSA: Luis Uruñuela, abogado, alcalde de Sevilla.

\section{LOS RESULTADOS}

A continuación, ofrezco al lector una serie de cuadros de datos para una más cómoda percepción de los resultados de estas elecciones al Parlamento Andaluz: 


\section{Cuadro 1}

DISTRIBUCION DE LOS VOTOS POR PARTIDO Y PROVINCIA

\begin{tabular}{|c|c|c|c|c|c|c|c|c|c|c|}
\hline & Almería & Cádiz & Córdoba & Granada & Huelva & Jaén & Málaga & Sevilla & Total & $\% *$ \\
\hline $\begin{array}{lllll}\text { PSOE } & \ldots & \ldots & \ldots & \ldots\end{array}$ & 78.906 & 210.693 & 182.975 & 183.559 & 96.856 & 172.423 & 112.098 & 357.109 & 1.494 .619 & 52,00 \\
\hline $\mathrm{AP} \quad \ldots \quad \ldots c c c c$ & 27.999 & 58.932 & 64.540 & 59.863 & 21.936 & 54.302 & 72.306 & 124.596 & 484.474 & 16,85 \\
\hline UCD $\ldots \ldots \ldots$ & 35.637 & 43.918 & 48.579 & 51.749 & 32.452 & 53.717 & 42.950 & 62.142 & 371.144 & 12,91 \\
\hline PCA $\ldots \ldots \ldots \ldots$ & 6.667 & 23.269 & 46.368 & 31.295 & 9.155 & 29.758 & 29.266 & 67.566 & 243.344 & 8,46 \\
\hline PSA $\ldots \ldots \ldots \ldots$ & 6.214 & 35.407 & 18.367 & 9.986 & 7.241 & 11.544 & 27.602 & 37.348 & 153.709 & 5,34 \\
\hline
\end{tabular}

* Sobre total votos emitidos.

Censo electoral: 4.346 .783 .

Votos emitidos: $2.874 .486(66,12$ por 100$)$.

Abstenciones: 1.472 .297 (33,87 por 100).

Votos nulos: 24.867 (0,86 por 100 sobre total votos emitidos).

Votos blancos: $9.327(0,32$ por 100 sobre total votos emitidos). 
Cuadro 2

\section{DIPUTADOS CONSEGUIDOS POR CADA PARTIDO}

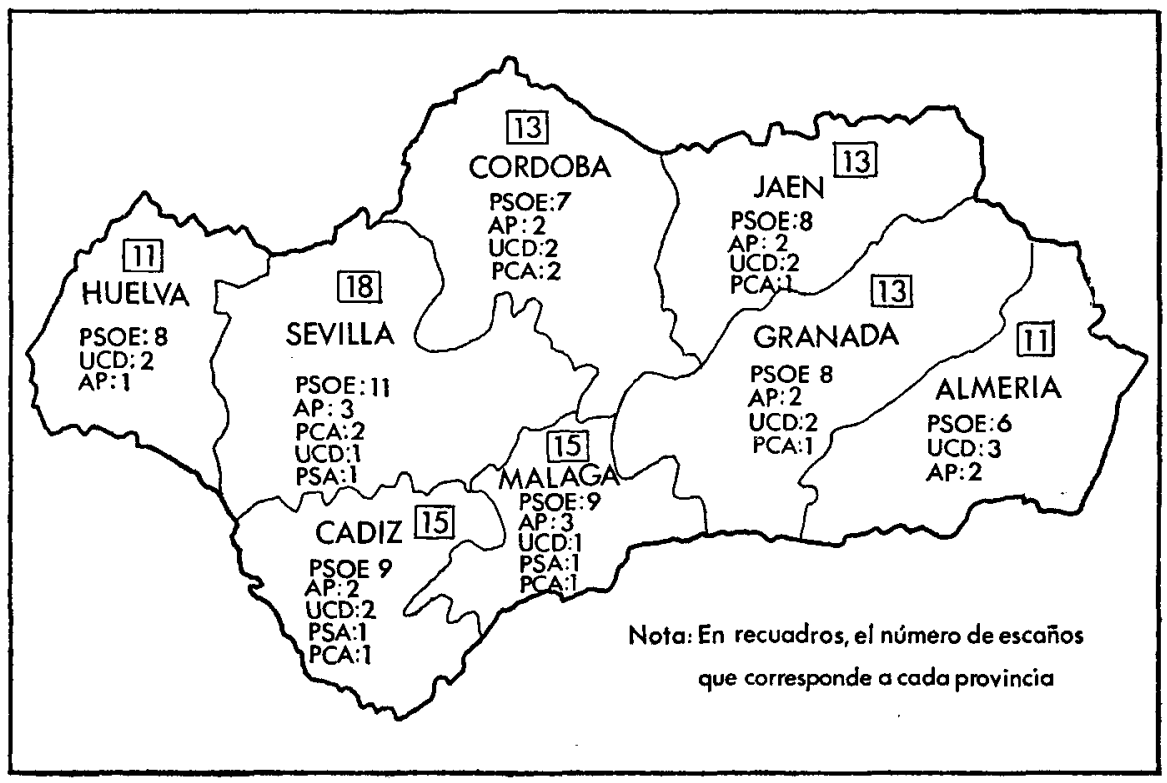




\title{
RESULTADOS PROVINCIALES
}

\author{
Cuadro $3 a$
}

ALMERIA

Número de secciones: 340

Número de mesas: 423

Censo electoral: 276.311

\begin{tabular}{|c|c|c|c|c|c|}
\hline & $\begin{array}{l}\text { N.o de votos en } \\
\text { ciudades de } \\
\text { menos de } 20.000 \\
\text { habitantes }\end{array}$ & $\begin{array}{l}\text { N. }{ }^{\circ} \text { de votos en } \\
\text { ciudades de } \\
\text { más de } 20.000 \\
\text { habitantes }\end{array}$ & Total votos & $\% *$ & Escaños \\
\hline $\begin{array}{lllll}\text { UCD } & \ldots & \ldots & \ldots & \ldots\end{array}$ & 26.908 & 8.729 & 35.637 & 21,82 & 3 \\
\hline $\mathrm{AP} \ldots \ldots \ldots \ldots \ldots$ & 13.481 & 14.158 & 27.999 & 17,15 & 2 \\
\hline PSOE $\ldots \ldots \ldots \ldots$ & 43.655 & 35.251 & 78.906 & 48,33 & 6 \\
\hline $\begin{array}{lllll}\mathrm{SI}^{1} & \ldots & \ldots & \ldots & \ldots\end{array}$ & 942 & 333 & 1.275 & 0,78 & \\
\hline $\mathrm{FN} \ldots \ldots \ldots \ldots$ & 1.888 & 1.272 & 3.160 & 1,93 & \\
\hline $\begin{array}{lllll}\text { PCA } & \ldots & \ldots & \ldots & \ldots\end{array}$ & 3.557 & 3.110 & 6.667 & 4,08 & \\
\hline $\mathrm{MCA}^{2} \ldots \ldots \ldots \ldots$ & 280 & 264 & 544 & 0,33 & \\
\hline 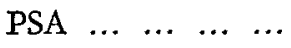 & 2.879 & 3.335 & 6.214 & 3,80 & \\
\hline $\mathrm{UCA}^{3} \ldots \ldots \ldots$ & 604 & 229 & 833 & 0,51 & \\
\hline
\end{tabular}

* Sobre total votos emitidos.

1 Sindicalistas Independientes.

2 Movimiento Comunista de Andalucía.

3 Unificación Comunista de Andalucía.

Votos a listas: 161.235 (98,76 por 100 sobre votos emitidos).

Votos nulos: 1.561 (0,95 por 100 sobre votos emitidos).

Votos blancos: 462 ( 0,28 por 100 sobre votos emitidos).

Votos emitidos: 163.258 (59,08 por 100 sobre censo electoral).

Abstenciones: 113.053 (40,91 por 100 sobre censo electoral). 


\section{Cuadro $3 b$}

CADIZ

Número de secciones: 680

Número de mesas: 913

Censo electoral: 641.499

\begin{tabular}{|c|c|c|c|c|c|}
\hline & $\begin{array}{l}\text { N.o de votos en } \\
\text { ciudades de } \\
\text { menos de } 20.000 \\
\text { habitantes }\end{array}$ & $\begin{array}{l}\mathrm{N} .^{\circ} \text { de votos en } \\
\text { ciudades de } \\
\text { más de } 20.000 \\
\text { habitantes }\end{array}$ & Total votos & $\%$ & Escaños \\
\hline $\begin{array}{lllll}\text { PCA } & \ldots & \ldots & \ldots & \ldots\end{array}$ & 7.533 & 15.736 & 23.269 & 5,95 & 1 \\
\hline $\begin{array}{lllll}\mathrm{UCD} & \ldots & \ldots & \ldots & \ldots\end{array}$ & 15.263 & 28.655 & 43.918 & 11,24 & 2 \\
\hline $\begin{array}{lllll}\operatorname{PST}^{1} & \ldots & \ldots & \ldots & \ldots\end{array}$ & 1.328 & 4.453 & 5.781 & 1,48 & \\
\hline $\begin{array}{lllll}\text { PSA } & \ldots & \ldots & \ldots & \ldots\end{array}$ & 3.803 & 31.604 & 35.407 & 9,06 & 1 \\
\hline $\operatorname{PSOE} \ldots \ldots$ & 52.352 & 158.341 & 210.693 & 53,95 & 9 \\
\hline $\mathrm{AP} \ldots \ldots \ldots \ldots$ & 7.468 & 51.464 & 58.932 & 15,09 & 2 \\
\hline $\mathrm{UCA}^{2} \ldots \ldots \ldots$ & 310 & 1.045 & 1.355 & 0,34 & \\
\hline PCM $(\mathrm{M}-\mathrm{L})^{3} \ldots \ldots$ & 240 & 947 & 1.187 & 0,30 & \\
\hline $\mathrm{FN} \ldots \ldots \ldots \ldots$ & 508 & 2.114 & 2.622 & 0,67 & \\
\hline $\mathrm{MFA}^{4} \ldots \ldots \ldots$ & 124 & 489 & 613 & 0,15 & \\
\hline$M{ }^{\prime} A^{5} \ldots \ldots \ldots \ldots$ & 176 & 978 & 1.154 & 0,29 & $\cdot$ \\
\hline
\end{tabular}

* Sobre total votos emitidos.

1 Partido Socialista de los Trabajadores.

2 Unificación Comunista de Andalucía.

3 PCE Marxista-Leninista.

4 Movimiento Falangista de Andalucía.

5 Movimiento Comunista de Andalucía.

Votos a listas: 384.931 (98,57 por 100 -sobre votos emitidos).

Votos nulos: 4.007 (1,02 por 100 sobre votos emitidos).

Votos blancos: 1.554 ( 0,39 por 100 sobre votos emitidos).

Votos emitidos: 390.492 (60,87 por 100 sobre censo).

Abstenciones: 251.007 (39,12 por 100 sobre censo). 
Cuadro $3 \mathrm{c}$

\section{CORDOBA}

Número de secciones: 600

Número de mesas: 689

Censo electoral: 506.875

\begin{tabular}{|c|c|c|c|c|c|}
\hline & $\begin{array}{l}\text { N.o de votos en } \\
\text { ciudades de } \\
\text { menos de } 20.000 \\
\text { habitantes }\end{array}$ & $\begin{array}{l}\text { N.o de votos en } \\
\text { ciudades de } \\
\text { más de } 20.000 \\
\text { habitantes }\end{array}$ & Total votoș & $\% *$ & Escaños \\
\hline $\begin{array}{lllll}\operatorname{PCA} & \ldots & \ldots & \ldots & \ldots\end{array}$ & 24.582 & 21.786 & 46.368 & 12,33 & 2 \\
\hline PSOE $\ldots \ldots \ldots \ldots$ & 87.283 & 95.692 & 182.975 & 48,66 & 7 \\
\hline 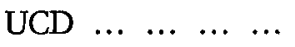 & 27.843 & 20.736 & 48.579 & 12,92 & 2 \\
\hline $\begin{array}{lllll}\text { PSA } & \ldots & \ldots & \ldots & \ldots\end{array}$ & 7.606 & 10.761 & 18.367 & 4,88 & \\
\hline $\mathrm{AP} \ldots \ldots \ldots \ldots$ & 22.636 & 41.904 & 64.540 & 17,16 & 2 \\
\hline $\mathrm{UCA}{ }^{1} \ldots \ldots \ldots$ & 570 & 544 & 1.114 & 0,29 & \\
\hline $\begin{array}{llllll}\mathrm{PST}^{2} & \ldots & \ldots & \ldots & \ldots\end{array}$ & 2.131 & 2.468 & 4.599 & 1,22 & \\
\hline $\operatorname{PCE}(M-L)^{3} \ldots \ldots$ & 336 & 394 & 730 & 0,19 & \\
\hline $\mathrm{MCA}^{4} \ldots \ldots \ldots$ & 322 & 483 & 805 & 0,21 & \\
\hline $\mathrm{FN} \ldots \ldots \ldots \ldots$ & 2.247 & 1.743 & 3.990 & 1,06 & \\
\hline FE-JONS $\ldots \ldots \ldots$ & 303 & 387 & 690 & 0,18 & \\
\hline
\end{tabular}

- Sobre total votos emitidos.

1 Unificación Comunista de Andalucía.

2 Partido Socialista de los Trabajadores.

3 PCE Marxista-Leninista.

4 Movimiento Comunista de Andalucía.

Votos a listas: 372.757 ( 99,14 por 100 sobre votos emitidos).

Votos nulos: 2.194 (0,58 por 100 sobre votos emitidos).

Votos blancos: 1.032 ( 0,27 por 100 sobre votos emitidos).

Votos emitidos: 375.983 ( 74,17 por 100 sobre censo).

Abstenciones: 130.892 (25,82 por 100 sobre censo). 
Cuadro 3d

GRANADA

Número de secciones: 656

Número de mesas: 660

Censo electoral: 525.385

\begin{tabular}{|c|c|c|c|c|c|}
\hline & $\begin{array}{l}\text { N.o de votos en } \\
\text { ciudades de } \\
\text { menos de } 20.000 \\
\text { habitantes }\end{array}$ & $\begin{array}{l}\text { N.o de votos en } \\
\text { ciudades de } \\
\text { más de } 20.000 \\
\text { habitantes }\end{array}$ & Total votos & $\%$ & Escaños \\
\hline $\operatorname{CGT}^{1} \ldots \ldots \ldots$ & 1.269 & 1.159 & 2.428 & 0,68 & \\
\hline $\mathrm{AP} \ldots \ldots \ldots$ & 23.269 & 36.594 & 59.863 & 16,95 & 2 \\
\hline $\begin{array}{lllll}\mathrm{UCD} & \ldots & \ldots & \ldots & \ldots\end{array}$ & 33.926 & 17.823 & 51.749 & 14,65 & 2 \\
\hline $\begin{array}{lllll}\operatorname{PSOE} & \ldots & \ldots & \ldots & \ldots\end{array}$ & 95.263 & 88.296 & 183.559 & 51,98 & 8 \\
\hline 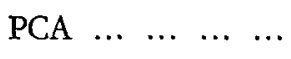 & 24.362 & 6.933 & 31.295 & 8,86 & 1 \\
\hline $\begin{array}{lllll}\text { PSA } & \ldots & \ldots & \ldots & \ldots\end{array}$ & 4.666 & 5.320 & 9.986 & 2,82 & \\
\hline $\mathrm{UCA}^{2} \ldots \ldots \ldots$ & 652 & 439 & 1.091 & 0,30 & \\
\hline $\begin{array}{lllll}\text { FE-JONS } & \ldots & \ldots & \ldots\end{array}$ & 912 & 427 & 1.339 & 0,37 & \\
\hline$M E^{3} \ldots \ldots \ldots$ & 302 & 178 & 480 & 0,13 & \\
\hline $\mathrm{LCR}^{4} \ldots \ldots \ldots c c$ & 一 & 一 & 一 & 一 & \\
\hline $\operatorname{MCA}^{5} \ldots \ldots \ldots$ & 331 & 376 & 707 & 0,20 & \\
\hline $\begin{array}{lllll}\mathrm{FN} & \ldots & \ldots & \ldots & \ldots\end{array}$ & 3.594 & 3.322 & 6.916 & 1,95 & \\
\hline
\end{tabular}

* Sobre total votos emitidos.

1 Candidatura Granadina de Trabajadores.

2 Unificación Comunista de Andalucía.

3 Movimiento Falangista de Andalucía.

- Liga Comunista Revolucionaria (trotskista).

5 Movimiento Comunista de Andalucía.

Votos a listas: 349.413 ( 98,95 por 100 sobre votos emitidos).

Votos nulos: $2.616(0,74$ por 100 sobre votos emitidos).

Votos blancos: 1.085 ( 0,30 por 100 sobre votos emitidos).

Votos emitidos: 353.114 (67,21 por 100 sobre censo).

Abstenciones: 172.271 (32,78 por 100 sobre censo). 


\author{
Cuadro 3e
}

\title{
HUELVA
}

Número de secciones: 321.

Número de mesas: 409.

Censo electoral: 293.223 .

\begin{tabular}{|c|c|c|c|c|c|}
\hline & $\begin{array}{l}\text { N.o de votos en } \\
\text { ciudades de } \\
\text { menos de } 20.000 \\
\text { habitantes }\end{array}$ & $\begin{array}{l}\text { N.० de votos en } \\
\text { ciudades de } \\
\text { más de } 20.000 \\
\text { habitantes }\end{array}$ & Total votos & $\% *$ & Escaños \\
\hline $\operatorname{LCR}^{1} \ldots \ldots \ldots$ & 301 & 92 & 393 & 0,22 & \\
\hline $\mathrm{MCA}^{2} \ldots \ldots \ldots \ldots$ & 547 & 127 & 674 & 0,38 & \\
\hline $\begin{array}{lllll}P S A & \ldots & \ldots & \ldots & \ldots\end{array}$ & 4.107 & 3.134 & 7.241 & 4,13 & \\
\hline $\begin{array}{lllll}\mathrm{UCD} & \ldots & \ldots & \ldots & \ldots\end{array}$ & 26.595 & 5.857 & 32.452 & 18,52 & 2 \\
\hline$M{ }^{3}{ }^{3} \ldots \ldots \ldots$ & 225 & 102 & 327 & 0,18 & \\
\hline $\mathrm{UCA}^{4} \ldots \ldots \ldots$ & 627 & 156 & 783 & 0,44 & \\
\hline $\begin{array}{lllll} & P C A & \ldots & \ldots & \ldots\end{array}$ & 7.303 & 1.852 & 9.155 & 5,22 & \\
\hline $\begin{array}{lllll}\operatorname{PST}^{5} & \ldots & \ldots & \ldots & \ldots\end{array}$ & 730 & 219 & 949 & 0,54 & \\
\hline $\mathrm{AP} \ldots \ldots \ldots \ldots$ & 12.841 & 9.095 & 21.936 & 12,52 & 1 \\
\hline $\mathrm{FN} \ldots \ldots \ldots$ & 1.787 & 817 & 2.604 & 1,48 & \\
\hline $\operatorname{PSOE} \ldots \ldots \ldots$ & 69.651 & 27.205 & 96.856 & 55,28 & 8 \\
\hline
\end{tabular}

* Sobre total votos emitidos.

1 Liga Comunista Revolucionaria.

2 Movimiento Comunista de Andalucía.

3 Movimiento Falangista de Andalucía.

4 Unificación Comunista de Andalucía.

5 Partido Socialista de los Trabajadores.

Votos a listas: 173.370 (98,96 por 100 sobre votos emitidos).

Votos nulos: 1.304 (0,74 por 100 sobte votos emitidos).

Votos blancos: 512 (0,29 por 100 sobre votos emitidos).

Votos emitidos: 175.186 (59,74 por 100 sobre censo).

Abstenciones: 113.662 ( 38,76 por 100 sobre censo). 


\section{CuAdro $3 \mathrm{f}$}

\section{JAEN}

Número de secciones: 539.

Número de mesas: 781 .

Censo electoral: 448.297.

\begin{tabular}{|c|c|c|c|c|c|}
\hline & $\begin{array}{l}\text { N.o de votos en } \\
\text { ciudades de } \\
\text { menos de } 20.000 \\
\text { habitantes }\end{array}$ & $\begin{array}{l}\text { N.॰ de votos en } \\
\text { ciudades de } \\
\text { más de } 20.000 \\
\text { habitantes }\end{array}$ & Total votos & $\% *$ & Escaños \\
\hline $\begin{array}{lllll}U C D & \ldots & \ldots & \ldots & \ldots\end{array}$ & 40.155 & 13.562 & 53.717 & 16,09 & 2 \\
\hline $\begin{array}{lllll}\mathrm{PCA} & \ldots & \ldots & \ldots & \ldots\end{array}$ & 20.566 & 9.192 & 29.758 & 8,91 & 1 \\
\hline $\mathrm{AP} \ldots \ldots \ldots \ldots$ & 23.945 & 30.357 & 54.302 & 16,26 & 2 \\
\hline $\begin{array}{lllll}\operatorname{PSA} & \ldots & \ldots & \ldots & \ldots\end{array}$ & 4.911 & 6.633 & 11.544 & 3,45 & \\
\hline FE-JONS $\ldots \ldots$ & 471 & 308 & 779 & 0,23 & \\
\hline $\operatorname{MFA}^{\prime} \ldots \ldots \ldots$ & 227 & 185 & 412 & 0,12 & \\
\hline PSOE $\ldots \ldots \ldots$ & 104.344 & 68.079 & 172.423 & 51,65 & 8 \\
\hline $\operatorname{SPA}^{2} \ldots \ldots$ & 259 & 231 & 490 & 0,14 & \\
\hline $\mathrm{UCA}^{3} \ldots \ldots$ & 570 & 359 & 929 & 0,27 & \\
\hline $\mathrm{FN} \ldots \ldots \ldots$ & 3.626 & 2.652 & 6.278 & 1,88 & \\
\hline$M M^{4} \ldots \ldots \ldots$ & 259 & 296 & 555 & 0,16 & \\
\hline
\end{tabular}

* Sobre total votos emitidos.

1 Movimiento Falangista de Andalucia.

2 Solidaridad Popular Andaluza.

3 Unificación Comunista de Andalucía.

4 Movimiento Comunista de Andalucía.

Votos a listas: 331.187 (99,22 por 100 sobre votos emitidos).

Votos nulos: 1.992 ( 0,59 por 100 sobre votos emitidos).

Votos blancos: 608 ( 0,18 por 100 sobre votos emitidos).

Votos emitidos: 333.787 ( 74,45 por 100 sobre censo).

Abstenciones: 114.510 (25,54 por 100 sobre censo). 


\section{CUAdro $3 \mathrm{~g}$}

MALAGA

Número de secciones: 640.

Número de mesas: 710.

Censo electoral: 664.447.

\begin{tabular}{|c|c|c|c|c|c|}
\hline & $\begin{array}{l}\text { N.o de votos en } \\
\text { ciudades de } \\
\text { menos de } 20.000 \\
\text { habitantes }\end{array}$ & $\begin{array}{l}N .^{\circ} \text { de votos en } \\
\text { ciudades de } \\
\text { más de } 20.000 \\
\text { habitantes }\end{array}$ & Total votos & $\% *$ & Escaños \\
\hline $\mathrm{AP} \ldots \ldots \ldots \ldots$ & 15.799 & 56.507 & 72.306 & 17,98 & 3 \\
\hline $\mathrm{OC}(\mathrm{BR})^{1} \ldots \ldots \ldots$ & 5 & 5 & 10 & 0,002 & \\
\hline $\mathrm{FN} \ldots \ldots \ldots \ldots$ & 1.155 & 2.737 & 3.892 & 0,96 & \\
\hline $\begin{array}{lllll}\text { PCA } & \ldots & \ldots & \ldots & \ldots\end{array}$ & 13.868 & 15.398 & 29.266 & 7,27 & 1 \\
\hline $\mathrm{UCA}^{2} \ldots \ldots \ldots$ & 315 & 748 & 1.063 & 0,26 & \\
\hline $\begin{array}{lllll}\operatorname{PSA} & \ldots & \ldots & \ldots & \ldots\end{array}$ & 7.282 & 20.320 & 27.602 & 6,86 & 1 \\
\hline $\begin{array}{llllll}\operatorname{PSOE} & \ldots & \ldots & \ldots & \ldots\end{array}$ & 59.995 & 152.103 & 212.098 & 52,75 & 9 \\
\hline $\mathrm{UCD} \ldots \ldots$ & 16.185 & 26.765 & 42.950 & 10,68 & 1 \\
\hline $\operatorname{MFA}^{3} \ldots \ldots \ldots$ & 141 & 386 & 527 & 0,13 & \\
\hline $\operatorname{MCA}^{4} \ldots \ldots \ldots \ldots$ & 326 & 804 & 1.130 & 0,28 & \\
\hline FE-JONS $\ldots \ldots \ldots$ & 203 & 578 & 781 & 0,19 & \\
\hline $\operatorname{PCE}(M-L)^{5} \ldots \ldots$ & 254 & 600 & 854 & 0,21 & \\
\hline $\begin{array}{llll}\operatorname{PCOE}^{6} & \ldots & \ldots & \ldots\end{array}$ & 1.042 & 2.536 & 3.578 & 0,88 & \\
\hline
\end{tabular}

* Sobre total votos emitidos.

1 Organización Comunista (Bandera Roja).

2 Unificación Comunista de Andalucfa.

3 Movimiento Falangista de Andalucía.

4 Movimiento Comunista de Andalucía.

5 Partido Comunista de España (marxista-leninista).

6 Partido Comunista Obrero Español.

Votos a listas: 396.057 (98,50 por 100 sobre votos emitidos).

Votos nulos: 4.549 (1,13 por 100 sobre votos emitidos).

Votos blancos: 1.468 ( 0,36 por 100 sobre votos emitidos).

Votos emitidos: 402.074 (60,51 por 100 sobre censo).

Abstenciones: 262.373 (39,48 por 100 sobre censo). 
Cuadro $3 \mathrm{~h}$

SEVILLA

Número de secciones: 1.128 .

Número de mesas: 1.561 .

Censo electoral: 990.746 .

\begin{tabular}{|c|c|c|c|c|c|}
\hline & $\begin{array}{l}\mathrm{N} .{ }^{\circ} \text { de votos en } \\
\text { ciudades de } \\
\text { menos de } 20.000 \\
\text { habitantes }\end{array}$ & $\begin{array}{l}\text { N. }{ }^{\circ} \text { de votos en } \\
\text { ciudades de } \\
\text { más de } 20.000 \\
\text { habitantes }\end{array}$ & Total votos & $\% *$ & Escaños \\
\hline $\begin{array}{lllll}\text { UCD } & \ldots & \ldots & \ldots & \ldots\end{array}$ & 31.169 & 30.973 & 62.142 & 9,18 & 1 \\
\hline $\mathrm{OC}(\mathrm{BR})^{1} \ldots \ldots \ldots$ & 一 & 一 & 一 & 一 & \\
\hline $\begin{array}{lllll}\mathrm{AP} & \ldots & \ldots & \ldots & \ldots\end{array}$ & 35.139 & 89.457 & 124.596 & 18,42 & 3 \\
\hline $\operatorname{PCOE}^{2} \ldots \ldots$ & 1.675 & 2.638 & 4.313 & 0,63 & \\
\hline $\mathrm{UCA}^{3} \ldots \ldots \ldots$ & 418 & 535 & 953 & 0,14 & \\
\hline $\begin{array}{lllll}\text { PSA } & \ldots & \ldots & \ldots & \ldots\end{array}$ & 11.616 & 25.732 & 37.348 & 5,52 & 1 \\
\hline PSOE $\ldots \ldots \ldots \ldots$ & 123.960 & 233.149 & 357.109 & 52,80 & 11 \\
\hline $\begin{array}{llllll} & P C A & \ldots & \ldots & \ldots & \ldots\end{array}$ & 32.684 & 34.882 & 67.566 & 9,99 & 2 \\
\hline $\mathrm{FN} \ldots \ldots \ldots$ & 2.054 & 3.432 & 5.486 & 0,81 & \\
\hline $\operatorname{LCR}^{4} \ldots \ldots \ldots$ & 210 & 292 & 502 & 0,07 & \\
\hline $\operatorname{PST}^{5} \ldots \ldots$ & 1.815 & 2.405 & 4.220 & 0,62 & \\
\hline $\mathrm{MCA}^{6} \ldots \ldots \ldots \ldots$ & 636 & 476 & 1.112 & 0,16 & \\
\hline PCE $(M-L)^{7} \ldots \ldots$ & 279 & 537 & 816 & 0,12 & \\
\hline $\mathrm{MFA}^{8} \ldots \ldots \ldots \ldots$ & 337 & 467 & 804 & 0,11 & \\
\hline
\end{tabular}

* Sobre total votos emitidos.

1 Organización Comunista (Bandera Roja).

2 Partido Comunista Obrero Español.

3 Unificación Comunista de Andalucía.

4 Liga Comunista Revolucionaria.

5 Partido Socialista de los Trabajadores.

- Movimiento Comunista de Andalucía.

7 Partido Comunista de España (marxista-leninista).

8 Movimiento Falangista de Andalucía.

Votos a listas: 666.967 (98,63 por 100 sobre votos emitidos).

Votos nulos: 6.644 (0,98 por 100 sobre votos emitidos).

Votos blancos: 2.606 (0,38 por 100 sobre votos emitidos).

Votos emitidos: 676.217 (68,25 por 100 sobre censo).

Abstenciones: 314.529 (31,64 por 100 sobre censo). 
Cuadro 4

REPARTO DE ESCAÑOS EN EL PARLAMENTO ANDALUZ

TRAS LA JORNADA DEL 23 DE MAYO DE 1982

\begin{tabular}{|c|c|c|c|c|c|c|c|c|c|}
\hline & Almería & Cádiz & Córdoba & Granada & Huelva & Jaén & Málaga & Sevilla & Total \\
\hline PSOE $\ldots \ldots \ldots$ & 6 & 9 & 7 & 8 & 8 & 8 & 9 & 11 & 66 \\
\hline $\mathrm{AP} \ldots \ldots \ldots$ & 2 & 2 & 2 & 2 & 1 & 2 & 3. & 3 & 17 \\
\hline UCD $\ldots \ldots$ & 3 & 2 & 2 & 2 & 2 & 2 & 1 & 1 & 15 \\
\hline PCA $\ldots \ldots$ & & 1 & 2 & 1 & & 1 & 1 & 2 & 8 \\
\hline $\begin{array}{lllll}P S A & \ldots & \ldots & \ldots\end{array}$ & & 1 & & & & & 1 & 1 & 3 \\
\hline Total ... ... & 11 & 15 & 13 & 13 & 11 & 13 & 15 & 18 & 109 \\
\hline
\end{tabular}


CuAdro 5

RESULTADOS DE LAS ANTERIORES ELECCIONES EN ANDALUCIA

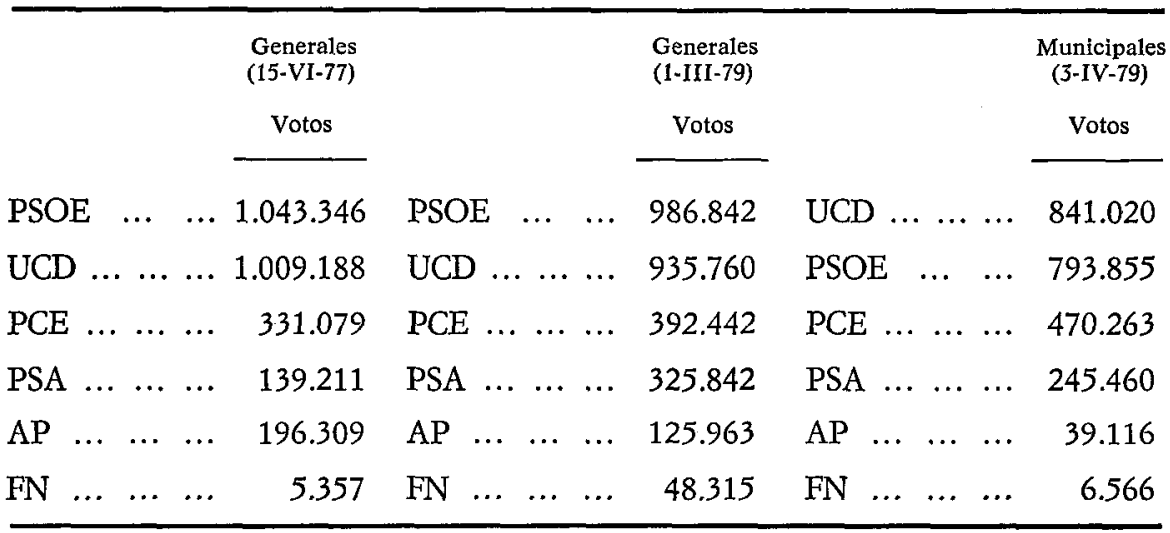

Cuadro 6

EVOLUCION DE LA ABSTENCION EN ANDALUCIA

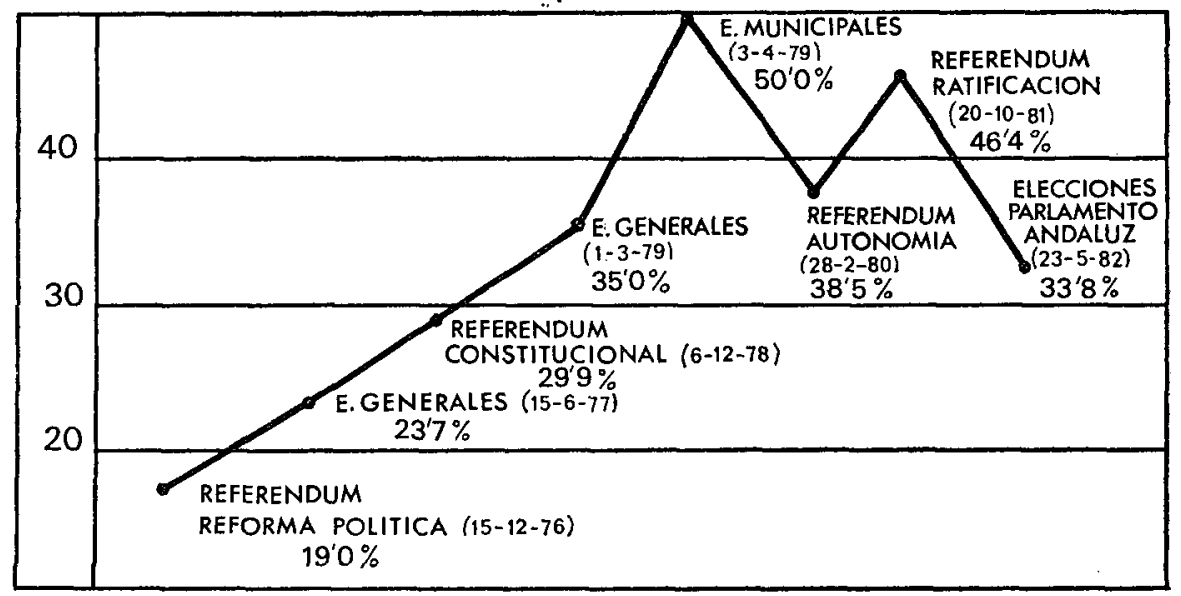


A la vista de los cuadros de datos anteriormente expuestos, se podrían extraer varias conclusiones:

1. ${ }^{\mathrm{a}}$ El PSOE consigue, en las primeras elecciones al Parlamento Andaluz, la más arrolladora victoria que fuerza o coalición política alguna haya alcanzado tras la restauración de la democracia en España (52,0 por 100 de los votos emitidos).

2. $\quad$ El PSOE ha afianzado su voto en las zonas urbanas y rurales, atrayéndose hacia sus posiciones ideológicas aproximadamente a la mitad de los electorados comunista y andalucista del PSA (PCA: 470.263 votos en las municipales de abril de $1979,243.344$ en estas elecciones; PSA: 245.460 votos en las municipales, 153.709 en estas elecciones).

3. ${ }^{\mathrm{a}} \mathrm{La}$ crisis del PCE ha llegado también a Andalucía, donde los comunistas han sufrido su mayor descalabro electoral desde 1977.

4. ${ }^{\text {a }}$ El confusionismo ideológico ha deparado al PSA su casi desaparición del espectro político andaluz. Otro motivo de peso para la explicación del derrumbamiento andalucista lo constituyen las crisis habidas en el seno del partido al tratar su dirección política de conseguir a toda costa la alcaldía de Sevilla tras las municipales, desechando las ventajosas posiciones alcanzadas en Granada y Huelva. Se da la circunstancia de que el «partido andaluz» tiene actualmente más escaños en el Congreso de los Diputados de Madrid que en el Parlamento Andaluz.

5. ${ }^{\text {a }}$ La fuerte subida de Alianza Popular - sobre todo en los grandes núcleos urbanos- se ve ensombrecida por el triunfo socialista. La derecha andaluza no ha resucitado tras el mensaje de Fraga. Alianza Popular ha arrebatado votos a $\mathrm{UCD}$, en efecto, pero ni los suficientes para pasar a ocupar el lugar del partido del gobierno en el mapa político andaluz, ni los necesarios para, uniéndolos a los centristas, formar el «gran bloque de derecha» reclamado por el líder aliancista Manuel Fraga (en las últimas elecciones generales de 1979 UCD obtuvo 935.760 votos; en estas elecciones los votos de UCD y AP juntos sólo alcanzan la cifra de 855.616). El electorado andaluz ha votado a la izquierda en un 60,46 por 100 (PSOE + PCA), y éste es el dato más significativo de estas elecciones.

6. ${ }^{a}$ Por último, señalar otro aspecto importante de esta consulta: la abstención no sólo ha sido frenada - en este caso concreto-, sino que habiendo alcanzado el techo del 50 por 100 en las elecciones municipales de 1979 , ha sido rebajada al 33,8 por 100 , similar a la cota alcanzada en el referéndum constitucional de 1978.

Fernando Ollero Butler 